\title{
VERTICAL SPINDLE COTTON HARVESTER WITH SYMMETRIC MOVEMENT OF SPINDLE DRUMS
}

\author{
Abdusalam Abdukarimov \\ Institute of Mechanics and Seismic Stability of Structures named after M.T. Urazbaev, Academy of Sciences of the \\ Republic of Uzbekistan, 31 Durmon Street, and 100125 Tashkent, Uzbekistan \\ Corresponding author: Abdusalam Abdukarimov, aabdusalam54@gmail.com
}

\begin{abstract}
The article deals with the trends in the development of structures, research work on modernization and creation of new vertical spindle cotton harvesting apparatus (CHA). Authors developed new CHA that works as follows: the CHA with a cotton picker moves on the cotton field; the cotton plants getting into the inter-drum slots are processed, that is, the raw cotton be harvested. The CHA are in their original position before picking raw cotton. When a thick cotton plant gets into the inter-drum slot of the front pair of drums, the spindle drums move apart and the inter-drum slot opens, while the connecting rods and the sliders move forward, providing the symmetrical opening of the slot relative to the longitudinal line of the cotton plant row. Further, this thick plant falls into the inter-drum slot of the second pair of drums, while the inter-drum slot of the second drums opens, while the connecting rods and the sliders move along the guides forward, providing the symmetrical opening of the slot relative to the longitudinal line of the cotton plant rows. With such a pairwise symmetric movement of the SD, depending on the thickness of the cotton plant, the force of spindle pressing on the cotton plant from both sides is identical and symmetrical, since the SD move symmetrically to the longitudinal line passing along the cotton plant row.
\end{abstract}

Key words: cotton, spindle, cotton picker, cotton harvester, drum, symmetry

\section{INTRODUCTION}

A cotton harvester with a symmetrical movement of the centers of rotation of the spindle drums was developed in the laboratory "Theory of Mechanisms and Machines" (TMM) of the Institute of Mechanics and Seismic Stability of Structures named after M.T. Urazbayev AS RUz [1]. The study relates to the machine-building industry, in particular, to agricultural harvesting machines, namely machines for raw cotton harvesting. The harvester contains four spindle drums (SD) installed in pairs to form a working slot, which bears the working bodies (spindles). During the execution of the technological process (raw cotton picking), the size of the slots between the SD in each pair of SD (front and rear pair of SD) changes depending on the thickness of the processed cotton bushes. The coincidence of the longitudinal line of the cotton bush row with the longitudinal line passing in the middle of the axes of the SD rotation is very important.

\section{REVIEW AND ANALYSIS OF EXISTING COTTON HARVESTING APPARATUS}

A cotton harvesting apparatus (CHA) is a wellknown unit; it includes four SD, installed in left and right frames - two SD in each frame; one of the frames is rigidly fixed on a stationary bar, and the second frame is hinged on this bar using a doublehinged dragbar (Figure 1) [2,3]. The disadvantage of such a CHA is that one frame is rigidly fixed on the bed, and the second frame is hinged on the bed with a double-hinged dragbar; the changes in the SB slots (for example, expansion) occur due to the movement of one frame, hinged on the bed using a double-hinged dragbar. This leads to the fact that during the cotton picking, the cultivated cotton bushes deviate from the natural axis towards the frame fixed with the double-hinged dragbar when a bush of large volume gets in, or it deviates in the other direction when a bush is of small volume, that is, the coincidence of the line of the cotton bush row with the longitudinal line passing in the middle of the axes of the SD rotation is violated. This leads to a decrease in the efficiency of raw cotton picking by the spindles due to the deviation of cotton bushes from the natural axis since the pressing force of the spindles in the cotton bushes in different spindles drums is different. This circumstance leads to a decrease in the reliability and productivity of the machine $[1,2]$.

A CHA is known, which contains the SD installed with the formation of a working slot; the SD are 
placed in spring-loaded frames, moved by means of rollers on the guides (Figures 2, 3, 4) [4].

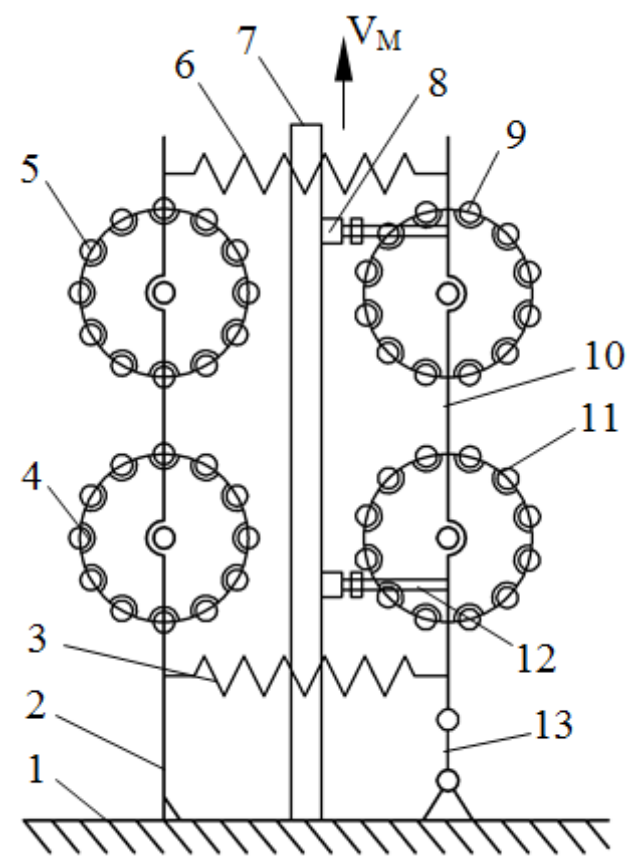

Fig. 1. Scheme of the existing cotton harvester: 1bar; 2 - fixed lever; 3,6 - springs; 4,5,9,11 - spindle drums; 7 - beam; 8.12 - support; 10 - movable lever (connecting rod); 13 - crank

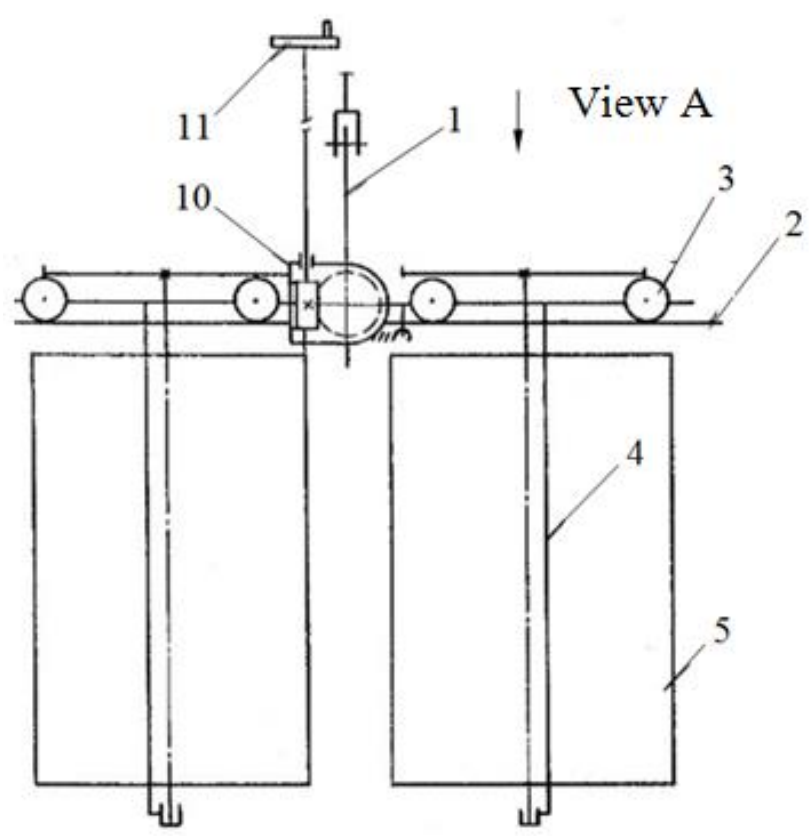

Fig. 2. Scheme of the cotton harvester, invention certificate No. 799690. 1 - skeleton; 2 - guide; 3 - roller; 4 - frame; 5 - spindle drum; 10 - screw; 11 - flywheel

The disadvantage of such a CHA is that the changes in the slot of one of the SD pairs cause a parallel expansion of the slots of the second SD pair, which, in turn, sharply reduces the efficiency of the raw cotton harvesting by the spindles of the second pair of spindle drums since in this case there is no pressing force from the sides of the SD on the cotton bushes.
This circumstance also leads to a decrease in the productivity of the machine.

Figure 5 shows a mechanism for adjusting the working slot of a cotton harvester [5], which includes a movable guide bar with wedges interacting through pushers with movable sections of spindle drums pressed by springs equipped with a tensioning device. This cotton picker has movable and fixed sections with installed spindle drums and is subjected to all the disadvantages of cotton harvesters described above, namely, the line of the cotton bush row and a longitudinal line running in the middle of the axes of spindle drum rotation are broken and the slot expansion of one of the pairs of spindle drums causes a parallel expansion of the slots of the second pair of spindle drums

The purpose of the design is to increase the efficiency of the CHA in raw cotton picking, to increase the productivity and reliability of the CHA and the entire cotton harvesting machine (CHM).

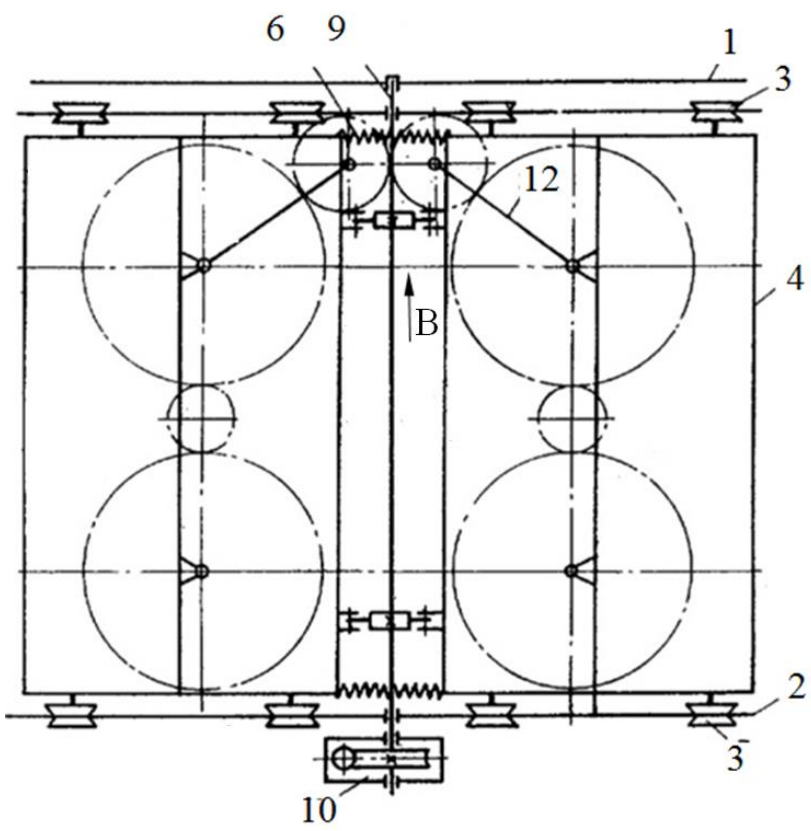

Fig. 3. View A. 1 - skeleton; 2 - guide; 3 - roller; 4 frame; 5 - spindle drum; 6 - spring; 9 - rotary shaft; 10 screw; 11 - flywheel; 12 - dragbar

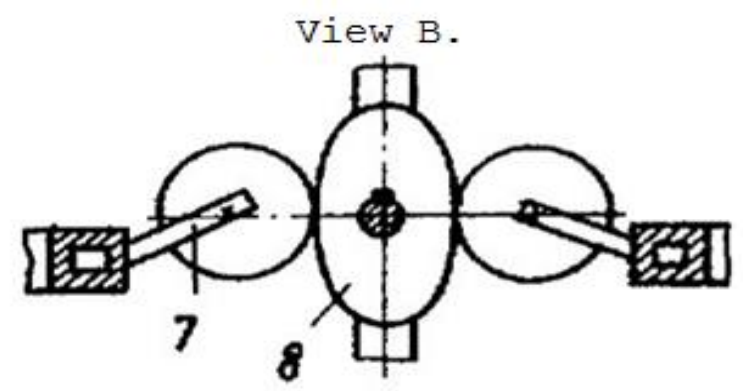

Fig. 4. View: B. 7 - roller; 8 - cam

For this, it is necessary during the raw cotton picking, to ensure that each pair of spindle drums independently moves apart symmetrically relative to 
the longitudinal line passing along the cotton bush row.

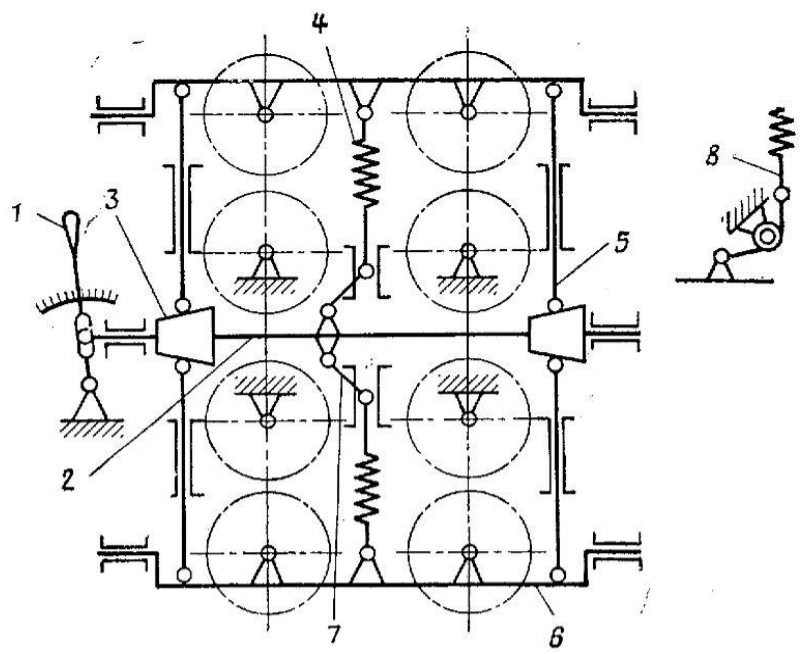

Fig. 5. Diagram of the cotton harvesting machine invention certificate No. 393998. 1 - lever; 2 - guide bar; 3 - wedge;

4 - spring; 5 - pusher; 6-frame; 7 - movable link; 8 flexible link

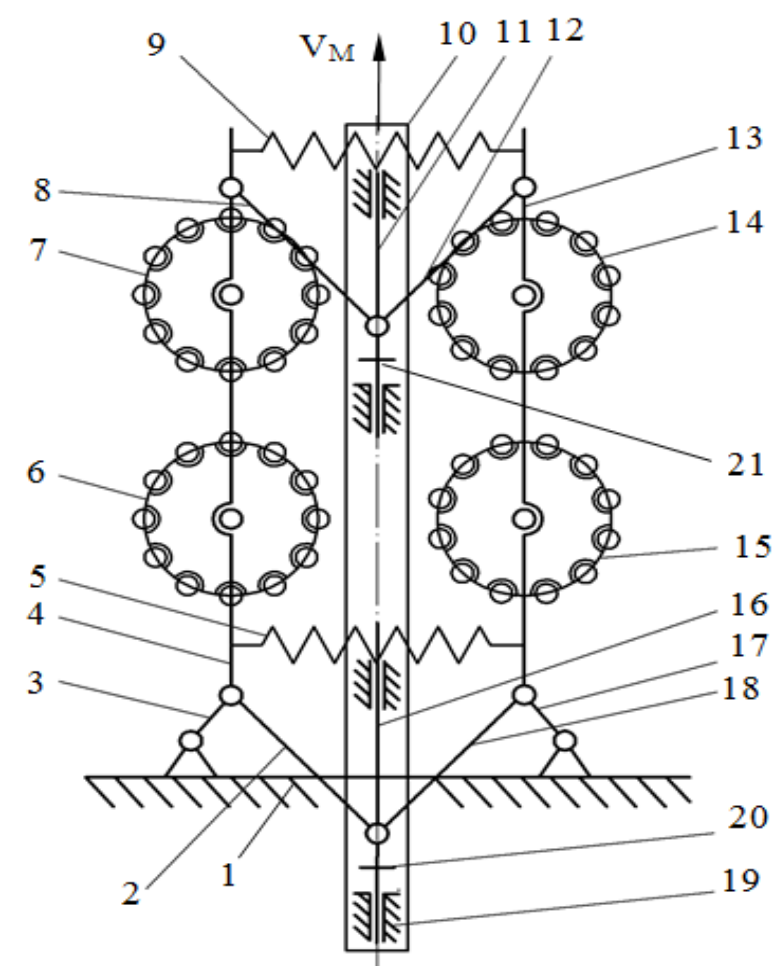

Fig. 6. Diagram of the new cotton harvester: 1 - bar; 2, 8, 12, 18 - connecting rod; 3, 17 - crank; 4, 13 - frame; 5, 9 spring; 6, 7, 14, 15 - spindle drums; 10 - beam; 11, 16 slider; 19 - guide

In this case, the spindles of each pair of spindle drums are pressed against the bushes of the harvested cotton with the same force necessary for picking cotton, and the longitudinal line of the cotton bush must always coincide with the longitudinal line passing through the center of the axes of the spindle drums. This goal is achieved by the fact that the CHA includes two frames fixed by means of cranks on a fixed bar with the sequential placement of pairs of vertical-spindle drums, and the ends of the frames are also pivotally connected to the fixed beam by means of connecting rods and sliders.

\section{COTTON HARVESTER WITH SYMMETRICAL MOVEMENT OF THE CENTERS OF ROTATION OF THE SPINDLE DRUM}

The technical solution is illustrated by the diagram in Figure 6. Figures 7, 8, 9, 10 show the spindle drums in different positions. The cotton harvester consists of a fixed bar 1 . Frames 4 and 13 are hinged to bar 1 with the cranks 3 and 17, spindle drums 6,7 and 14 , 15 are sequentially placed on the frames with the ability to rotate around their axes. The frames are hinged at both ends with sliders 11 and 16 using rods 2, 8, 12 and 18. Sliders 11 and 16 have guides 19 installed in beam 10 , which is rigidly perpendicularly connected to beam 1 . The left and right parts of the cotton harvester are arranged symmetrically with respect to the axis passing along sliders 11 and 16, which coincides with the longitudinal axis of the cotton bushes. The left and right frames are tightened to each other by springs 5 and 9 . Slides 11 and 16 have stop blocks 20 and 21 .

The cotton harvesting apparatus works as follows: A cotton harvesting apparatus with a cotton harvester moves across the cotton field; cotton bushes falling into the inter-drum slots are processed, that is, raw cotton is picked. Before the start of the raw cotton picking, the cotton harvesting apparatus is in the position shown in Figure 7. When a thick cotton bush falls into the inter-drum slot of the front pair of drums 7 and 14, the spindle drums move apart and the interdrum slot opens, while connecting rods 8 and 12 together with slider 11 moving ahead, provide the opening of the slot symmetrically relative to the longitudinal line of the cotton bush row. Then, the cotton harvesting apparatus takes the position shown in Figure 8. Further, this thick cotton bush falls into the inter-drum slot of the second pair of drums 6 and 15 , the inter-drum slot of the second drums opens and connecting rods 2 and 18 , together with slider 16 , move ahead along the guides to provide for a symmetrical opening of the slot relative to the longitudinal line of the cotton bush row. In this case, the cotton harvesting apparatus can take the position indicated in Figure 8, if after a cotton bush of great thickness, a bush of the same great thickness falls into the inter-drum slot. Or, the CHA can take the position indicated in Figure 10, if after a cotton bush of large thickness, a cotton bush of a smaller thickness falls into the inter-drum slot, etc. Thus, with cranks 3 and 17, sliders 11 and 16 and connecting rods $2,8,12$ and 18 , the spindle drums 6,7 and 14, 15 move symmetrically in pairs. 
With such a pairwise symmetric movement of the spindle drums, depending on the thickness of the cotton bush, the pressing force of the spindles on the cotton bush from both sides is the same, and symmetrical since the spindle drums move symmetrically relative to the longitudinal line passing along the cotton bush rows.

An economic effect will be obtained as a result of the application of the proposed CHA; the effect is formed by increasing the completeness of raw cotton picking, reducing the raw cotton falling to the ground, increasing the CHA reliability and labor productivity, by ensuring a symmetrical and uniform pressure of cotton bushes with spindle drums, depending on the thickness of cotton bushes.

\section{INTER-DRUM TRANSMISSION OF THE CHA}

For normal operation of the vertical spindle CHA, the spindles of adjacent drums must be staggered [6]. In the process of picking raw cotton by the existing $\mathrm{CHA}$, the staggered arrangement of the spindles of adjacent spindle drums is regulated by special rulers. However, this regulated staggered arrangement of the spindles with a change in the center distance of the spindle drums is violated. This circumstance leads to a deterioration in the process of raw cotton picking, and leads to premature failure of the spindle supports due to the impact of the supports of adjacent spindles to each other. Figure 11 shows a diagram of the existing CHA with the inter-drum gear-lever differential transmission mechanism.

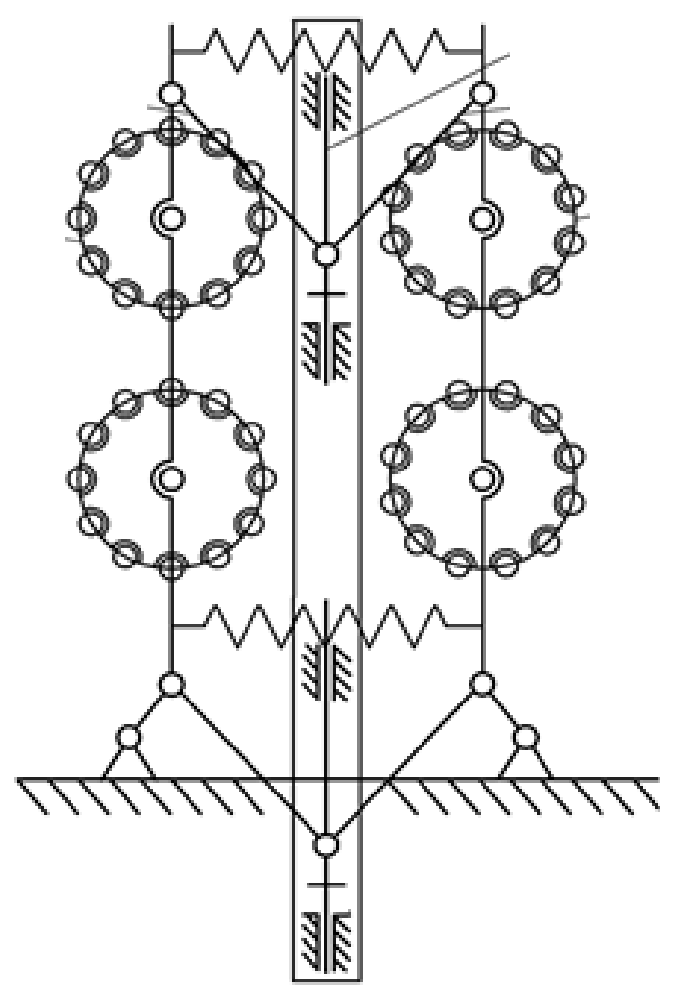

Fig. 7. Starting position

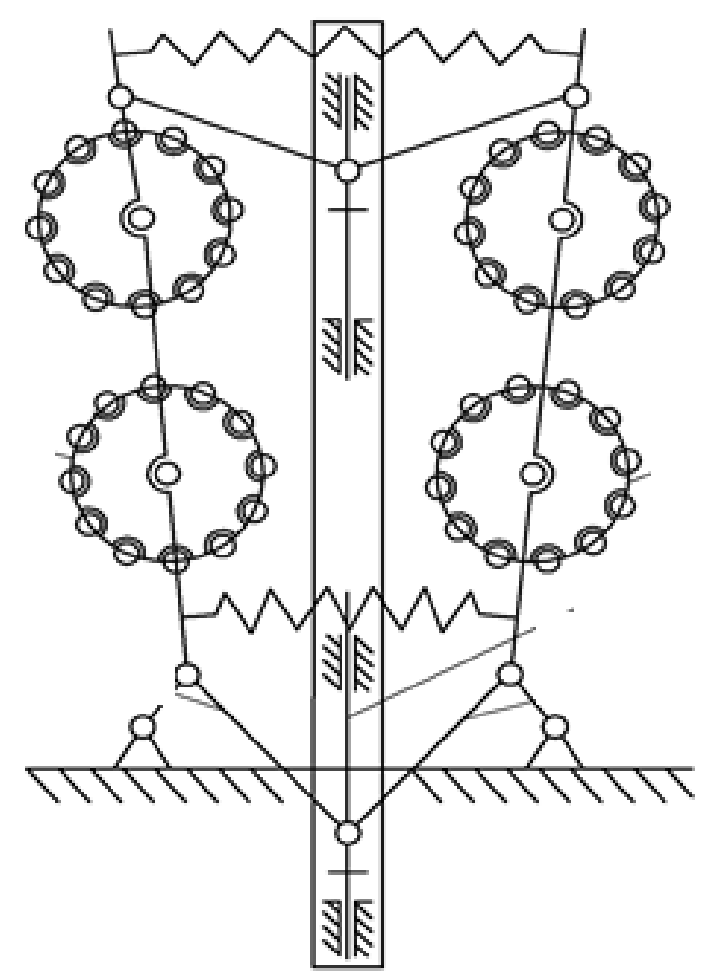

Fig. 8. Opening position of the first pair of spindles

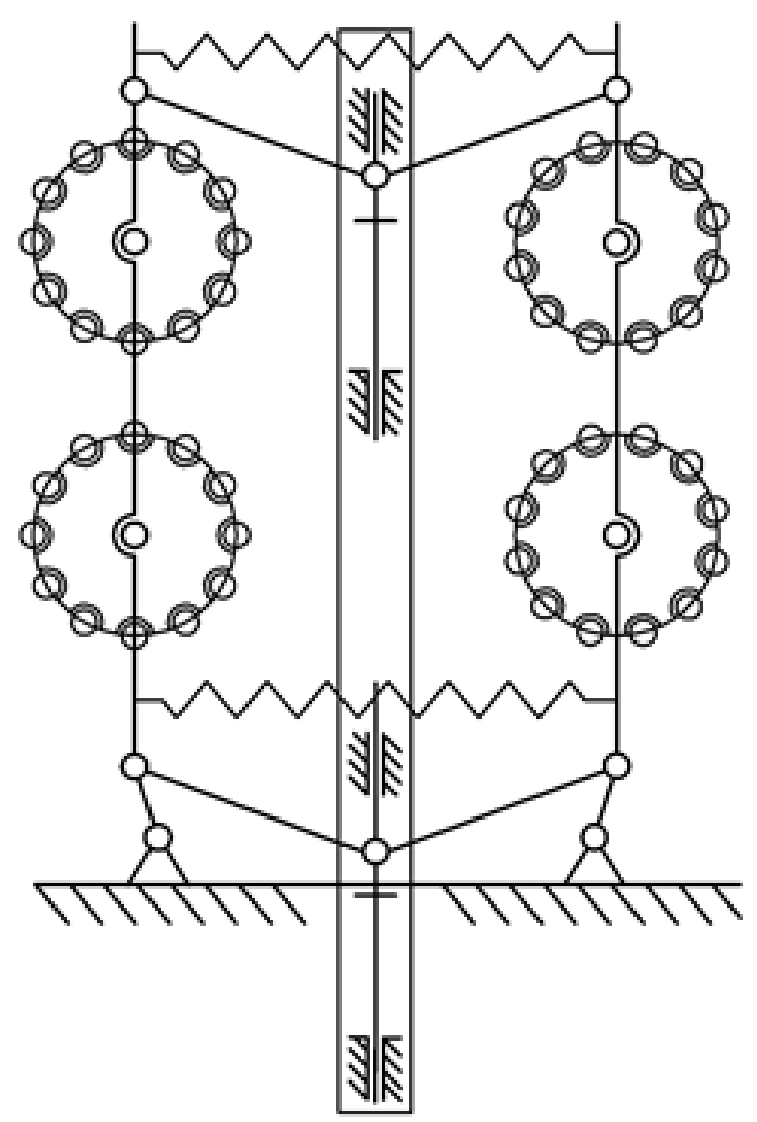

Fig. 9. Opening position of both pairs of spindles 


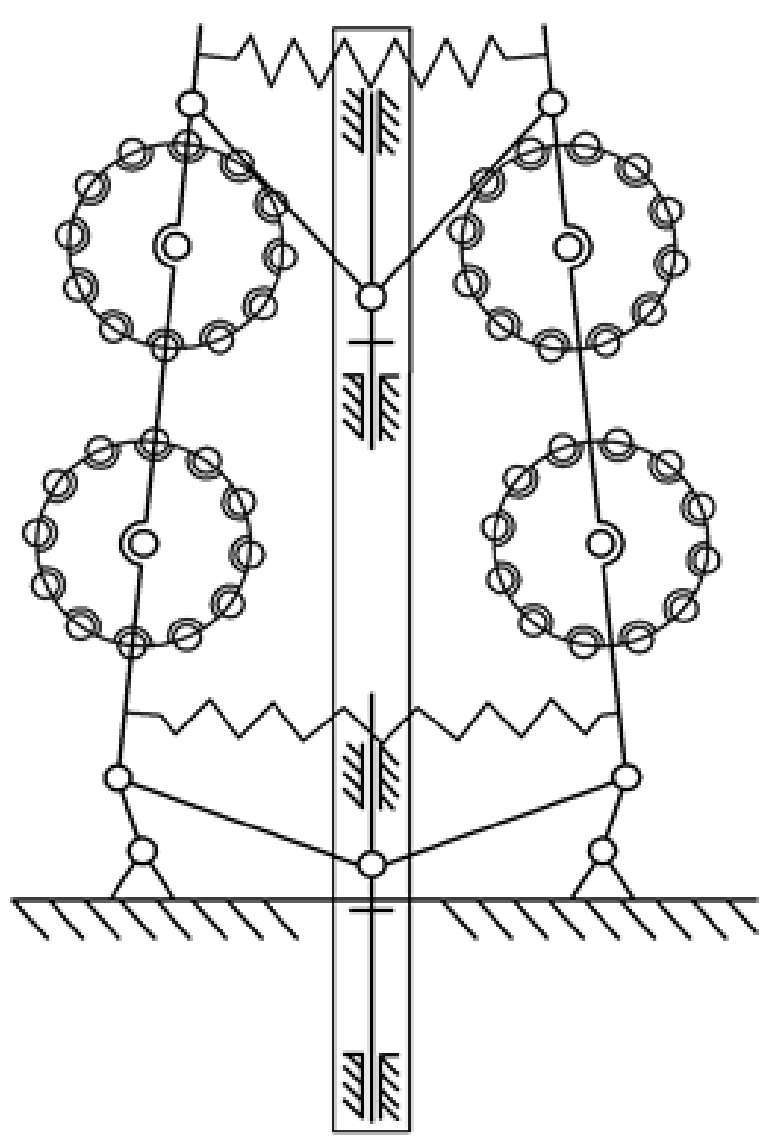

Fig.10. Opening position of the second pair of spindles

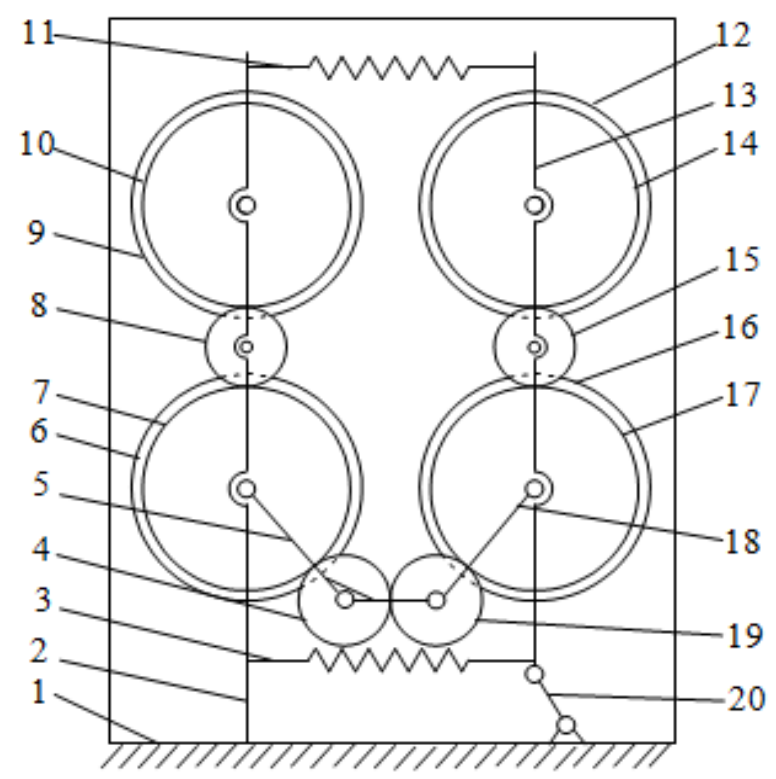

Fig. 11. Diagram of the existing cotton harvester with a gear-lever transmission mechanism. 1 - bar; 2 - fixed lever; 3.11 - spring; 4, 8, 15, 19 - intermediate gear wheels; 5, 13, 18 - movable arm; 6, 9, 12, 16 - spindle drums; 7, 10, 14, 17 - gear wheels; 20 - crank

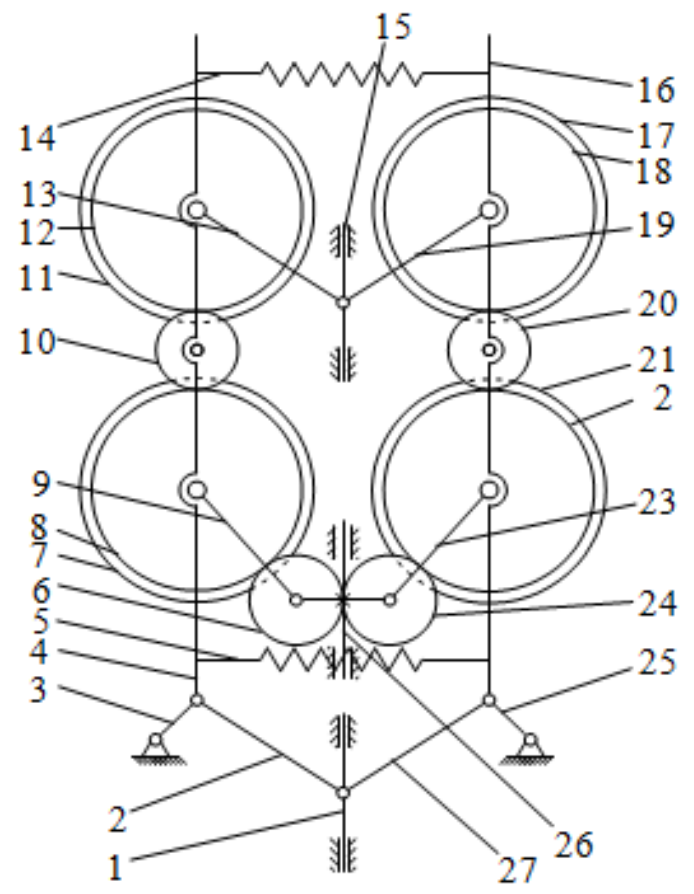

Fig. 12. Diagram of the CHA with symmetrical movement of the center of rotation of the working shafts. 1, 15 sliders; 2, 9, 13, 19, 23, 27 - levers; 3, 25 - rocker; 4, 16 movable bar; 5, 14 - spring; 6, 10, 20, 24 - intermediate gear wheels; 7, 11, 17, 21 - spindle drums; 8, 12, 18, 22 gear wheels; 26 - crosspiece

In the existing CHA, the inter-drum gear mechanism does not ensure the constancy of the gear ratios at the change in the center distance [7]. Our studies have shown that the use of other inter-drum transmission mechanisms in the existing CHA also does not provide synchronous rotation of the spindle drums [811]. So, there is no other solution to the problem except to change the roll modules (in this case, spindle drums) installation scheme on the bed [12]. The problem can be solved using the fourth module from the classification of roll modules as spindle drums [12] and the gear-lever differential mechanism we have invented [13].

Figure 12 shows a CHA with a symmetric arcuate movement of the centers of rotation of the spindle drums with a gear-lever differential transmission mechanism with a symmetric and arcuate movement of the centers of rotation of the driven and driving gears. Our studies [14] have shown that the CHA with symmetric and arcuate movement of the centers of rotation of the spindle drums paired with a gearlever differential transmission mechanism with symmetric and arcuate movement of the centers of rotation of the driven and driving gears ensures synchronous rotation of the spindle drums, regardless of the change in the center distance of the spindle drums. This prevents violations in the staggered arrangement of adjacent spindles. 


\section{CONCLUSIONS}

A cotton harvester was developed with a symmetric arcuate movement of the centers of rotation of the spindle drums, which prevents violations in the staggered arrangement of adjacent spindles. As a result of the application of the proposed CHA, an economic effect will be obtained, formed by increasing the completeness of raw cotton picking, reducing the raw cotton falling on the ground, increasing the reliability of the CHA and labor productivity, by ensuring a symmetric and uniform pressure on cotton bushes by spindle drums, depending on the thickness of the cotton bushes.

\section{REFERENCES}

1. Patent for invention, (2016), RUz No IAP 05158 Cotton harvesting apparatus Official Bulletin 2.

2. Markov M.N., Ladsman M.I., Davydov I.S., Butyrin A.V., Glazatov P.F., Iomdin N.G., Kutsenko V.K., (1958), J. Machines for cotton harvesting and ginning (Tashkent: Glavizdat Min. culture of the UzSSR).

3. Description of the invention certificate, (1965), SU 175773 Bul. 20.

4. Description of the invention certificate, (1981), SU 799690 Bul. 4.

5. Description of the invention certificate, (1973), SU 393998 Bul. 22.

6. Varyvdin V.V., Memetov R., Kuznetsov G.V., (1974), Analysis of the influence of errors in the dimensional chains of the drum drive on the accuracy of the staggered arrangement of spindles $\mathrm{J}$. Mechanization of cotton growing 31 .

7. Shekhovtsev V.M., Kuznetsov G.V., (1971), On errors in the installation of the staggered arrangement of the spindles of the cotton harvesting machine J. Mechanization of cotton growing 9-10.

8. Bahadirov G.A., Sultanov T.Z. and Abdukarimov A., (2020), Kinematic analysis of tooth-lever differential transmission mechanisms IOP Conf. Series: Earth and Environmental Science $\mathbf{6 1 4}$ doi:10.1088/1755-1315/614/1/012102.

9. Bahadirov G.A., Sultanov T.Z. and Abdukarimov A., (2020), Comparative analysis of two gear-lever differential inter-roller transmission mechanisms IOP Conf. Series: Earth and Environmental Science 614 doi:10.1088/1755-1315/614/1/012101.

10. Bahadirov G.A.,RavutovSh.T., Abdukarimov A. And Toshmatov E., (2021), Development of the methods of kinematic analysis of ellipticdrum of vertical-spindle cotton harvester IOP Conf. Series: Earth and Environmental Science 1030 doi:10.1088/1755-899X/1030/1/012160.

11. Abdukarimov A., (2016), Kinematic analysis of a gear-lever differential transmission mechanism with a symmetric, arcuate movement of the centers of rotation of the driven and driving gears relative to arbitrary symmetric centers J. of Problems in Mechanics 4 36-42.

12. Abdukarimov A., (2014), Classification of tworoll modules used in roller machines with variable center distance of the working shafts $\mathrm{J}$. Reports of AS RUz. 5 34-36.

13. Patent for invention RUz IAP 04934, (2014), Gear-lever transmission mechanism of roller machines (options) Option 3 Official Bulletin 8.

14. Abdukarimov A., (2016), Investigation of a gearlever differential transmission mechanism for roller machines with symmetric movement of the working shafts J. Bulletin of Tashkent State Technical University 3 18-23.

Received: March 12, 2021 / Accepted: December 20, 2021 / Paper available online: December 25, 2021 (C) International Journal of Modern Manufacturing Technologies 